\title{
The Processes of Violent Disintegration and Natural Creation of Matter in the Universe
}

\author{
Weitter Duckss \\ Independent Researcher, Zadar, Croatia \\ wduckss@gmail.com
}

\begin{abstract}
This article completes the circle of presenting the process of the constant growth of objects and systems and the topics to complete it consist of the visible matter violent disintegration and its re-creation inside the Universe. A constant process of the visible matter disintegration is presented as the end of the process, the proportions of which are gigantic, and the creation of the visible matter as the beginning of it. The disintegration of particles disturbs the balance of the Universe's wholeness; despite the enormous loss of the visible matter, the Universe is constantly growing. After having postponed it for a while, this article discusses the age of objects and the Universe as a consequence of the process of the constant matter growth. The acquired results are completely different from those, offered by the renowned experts of the time.
\end{abstract}

Keywords: disintegration of matter; particle formation; the age of the Universe

\section{Introduction}

The goal of the article is to unite the total processes of the constant matter growth inside the Universe, based on the independent research, the use of databases of generally accepted, easily verifiable evidence for the broadest community of readers. This article is a summary of the materials inside the process of the constant matter gathering, with the articles (Yukawa, 1935), (Duckss, 2019), (Duckss, 2018), and (Duckss, 2018) due to gravity or the law of universal gravitation.

The disintegration of matter is a process of turning the visible matter into the invisible matter and energy and it exists in the whole of the Universe. The loss of the enormous quantities of matter is replaced with the process of the visible matter constant growth out of the invisible matter inside the space or the whole of the Universe.

The age of the objects is analyzed through the time needed for matter to gather into dust, asteroids (comets) and increasingly larger objects, star systems, galaxies and finally the Universe.

\section{The Disintegration of Matter}

There are two stages of matter disintegration in the Universe. The first one is the disintegration of complex atoms and compounds into hydrogen. This process exists on Earth. The crust of Earth has more complex chemical composition than the melted interiority of Earth.

Table 1. The Earth crust and s mantle $\%$ crust of the Earth \% mantle of the Earth

$\begin{array}{lll}\mathrm{SiO}_{2} & 60,2 & 46 \\ \mathrm{Al}_{2} \mathrm{O}_{3} & 15,2 & 4,2 \\ \mathrm{CaO} & 5,5 & 3,2 \\ \mathrm{MgO} & 3,1 & 37,8 \\ \mathrm{FeO} & 3,8 & 7,5 \\ \mathrm{Na}_{2} \mathrm{O} & 3 & 0,4\end{array}$




$\begin{array}{lll}\mathrm{K}_{2} \mathrm{O} & 2.8 & 0,04 \\ \mathrm{Fe}_{2} \mathrm{O}_{3} & 2.5 & \\ \mathrm{H}_{2} \mathrm{O} & 1,4(1,1) & \\ \mathrm{CO}_{2} & 1,2 & \\ \mathrm{TiO}_{2} & 0,7 & \\ \mathrm{P}_{2} \mathrm{O}_{5} & 0,2 & \end{array}$

Table 1. comparison the chemical composition of the Earth crust and s mantle High temperatures of the melted interiority of Earth, when in contact with crust, water, air, etc., create an entire diapason of complex elements and compounds. Additional favorable conditions to create complex elements and compounds are the rotation around an axis (the differences in temperature between day and night), the changes of seasons and active geological processes.

An increment in both thermal radiation parameter and Eckert number results in decrement of temperature near the moving porous channel wall while it approaches to a zero in the region close to the boundary layer of the stationary channel wall" (Zigta, 2019)

High temperatures of the melted interiority of Earth disintegrate a part of complex elements and compounds into those that are simpler or less present. When temperature increases, the chemical composition of an object grows ever simpler and the last to exist are hydrogen and helium, while the rest make up to $2 \%$.

Table 2. Sun composition of the photosphere

$\begin{array}{cc}\text { Hydrogen } & 73,46 \% \\ \text { Helium } & 24,85 \% \\ \text { Oxygen } & 0,77 \% \\ \text { Carbon } & 0,29 \% \\ \text { Željezo } & 0,16 \% \\ \text { Neon } & 0,12 \% \\ \text { Nitrogen } & 0,09 \% \\ \text { Silicon } & 0,07 \% \\ \text { Magnesium } & 0,05 \% \\ \text { Sulfur } & 0,04 \%\end{array}$

Table 2. Sun composition of the photosphere (by mass) (John, 1979). Although the table 1 does not represent it, it is known that inside Earth, as well as on its crust, there are significant quantities of hydrogen-based compounds $\left(\mathrm{H}_{2} \mathrm{O}\right.$, hydrocarbons $\left.\mathrm{C}_{\mathrm{x}} \mathrm{H}_{\mathrm{x} . .}\right)$, there is no hydrogen on Mars, neither on its surface nor in the atmosphere, there is only „NASA again reported.. that Curiosity had detected a tenfold increase ('spike') in methane in the atmosphere around it in late 2013 and early 2014. Four measurements taken over two months in this period averaged 7 ppb, suggesting that methane is released at intervals“.

Table 3. The atmosphere of Mars

$\begin{array}{ll}95,97 \% & \text { carbon dioxide } \\ 1,93 \% & \text { argon } \\ 1,89 \% & \text { nitrogen } \\ 0,146 \% & \text { oxygen } \\ 0,0557 \% & \text { carbon monoxide } \\ 0,0210 \% & \text { water vapor } \\ 0,0100 \% & \text { nitrogen oxide }\end{array}$




$\begin{array}{ll}0,00025 \% & \text { neon } \\ 0,00008 \% & \text { hydrogen deuterium oxide } \\ 0,00003 \% & \text { krypton } \\ 0,00001 \% & \text { xenon }\end{array}$

Table 3. The composition by volume of the atmosphere of Mars (Williams, 2010). (The geological composition of the Mars surface: Mars is a terrestrial planet, consisting of the minerals of silicon and oxygen, metals and other elements that usually form rocks. The plagioclase feldspar NaAlSi3O8 to CaAl2Si2O8; pyroxenes are silicon-aluminium oxides with $\mathrm{Ca}, \mathrm{Na}, \mathrm{Fe}, \mathrm{Mg}, \mathrm{Zn}, \mathrm{Mn}$, Li replaced with $\mathrm{Si}$ and $\mathrm{Al}$; hematite $\mathrm{Fe} 2 \mathrm{O} 3$, olivine $(\mathrm{Mg}+2$, $\mathrm{Fe}+2) 2 \mathrm{SiO} 4 ; \mathrm{Fe} 3 \mathrm{O} 4$..)

The internal planets (just as Earth, hydrogen 0,00006\%) have only minor quantities of hydrogen in their atmospheres, due to the process of constant migration of hydrogen towards a more massive object (Sun).

Despite that fact, hydrogen (and helium) are constantly incoming into the atmosphere (it is estimated that the loss of hydrogen from Earth is $3 \mathrm{~kg} / \mathrm{s}$ and helium, $50 \mathrm{~g} / \mathrm{s}$ ) (Lagzi et al, 2013).

There are two options: either hydrogen was present in vast quantities on Earth long ago, or there exist the constant processes of hydrogen creation. The first option grows ever more incorrect, because of the fact that larger objects take hydrogen and helium from smaller objects, including Earth. That is obvious from the chemical composition of larger objects (such as Sun and gas giants) and the rest of smaller objects (with no exception).

Table 4. The atmosphere of Saturn and Titan moon

\section{Saturn}

$\begin{array}{ll}96,3 \pm 2,4 \% & \text { hydrogen }\left(\mathrm{H}_{2}\right) \\ 3,25 \pm 2,4 \% & \text { helium }(\mathrm{He}) \\ 0,45 \pm 0.2 \% & \text { methane }\left(\mathrm{CH}_{4}\right) \\ 0,0125 \pm 0,0075 \% & \text { ammonia }\left(\mathrm{NH}_{3}\right) \\ 0.0110 \pm 0,0058 \% & \text { hydrogen deuteride (HD) } \\ 0,0007 \pm 0,00015 \% & \text { ethane }\left(\mathrm{C}_{2} \mathrm{H}_{6}\right) \\ \text { Ices : } & \text { ammonia }\left(\mathrm{NH}_{3}\right) \\ & \text { water }\left(\mathrm{H}_{2} \mathrm{O}\right) \\ & \text { ammonium hydrosulfide }\left(\mathrm{NH}_{4} \mathrm{SH}\right)\end{array}$

In contrast to Saturn, Titan (Saturn's moon) has:

Stratosphere

$98,4 \%$

$1,4 \%$

$0,2 \%$

Lower troposphere:

$95,0 \%(97 \%)$

$1,4 \%(2.7 \pm 0.1 \%)$

$(0.1-0.2 \%)$

Table 4. Saturn and Titan (Saturn's moon) atmosphere (Williams, 2016), (Niemann, H. B. et al, 2005). Second stage, the existence of the process of disintegration or decomposition of matter is proved inside the small and large particle colliders. If the particles are influenced 
by the strong percussive force, then atoms (protons, electrons, neutrons) are decomposed after each collision into neutrinos and dark matter (invisible to our instruments).

Only in the process of the Sun's (as the object that emits waves) percussive waves to the atmosphere a significant quantity of matter gets disintegrated (some 10000 muons per $\mathrm{m}^{2}$ hits the surface of Earth every minute (the surface of Earth is $510072000 \mathrm{~km}^{2}$ )). (Street \& Stevenson, 1937).

In the period of $2,20 \times 10^{-6}$ of a second, muons are disintegrated into electrons and neutrinos:

$\mu^{-} \rightarrow \mathrm{e}^{-}+\underline{v_{\mathrm{e}}}+\underline{v_{\mu}}$

$\mu^{+} \rightarrow \underline{\mathrm{e}^{+}+v_{\mathrm{e}}+v_{\mu}}$ (Yukawa, 1935).

A chemical composition of atmosphere is the first to be exposed to the percussive waves (above $200 \mathrm{~km}$ ) consisting of the atomic oxygen $(\mathrm{O})$, helium $(\mathrm{He})$ and hydrogen $(\mathrm{H})$ (Duckss, 2019). It can be found out from the chemical composition of the outer atmosphere, which particles muons are created from. These are the particles that are exposed to the percussive waves first. The impact of the waves to the atmosphere (to the particles, the visible matter) also creates light, heat and ionizes particles (Duckss, 2018).

The probability density functions of the minimum and maximum order statistics of the transmuted logistic distribution are established and the relationships between the probability density functions of the minimum and maximum order statistics of the parent model and the probability density function of the transmuted logistic distribution are considered." (Samuel, 2019).

The disintegration of particles also takes place when two objects (asteroids, planets,...) collide. There is a significant disintegration of particles when objects fall into fast cyclones and also at fast rotating stars and when stars fall into fast rotating cyclones of the galactic centers. These cyclones are situated on the northern and southern poles of the gas giants, stars and galactic centers.

There is an infinite quantity of particles' collisions in the explosion of a star, percussive values of which are of the higher or even value as those in LHC. These collisions lead to the disintegration of large quantities of the star's mass (the most of its total mass).

To date it has been discovered (total number until today) just over 400 novae in the Milky Way. (Duckss, 2019).

Besides the disintegration of the larger part of its matter (depending on its power and type, it can be a complete or partial disintegration, with the rejection of a larger or smaller part of matter, when a core is preserved and it can make the rotation of the remainders around the axis to accelerate (a pulsar) or decelerate (a small red star)), a chemical composition of the remainders of a star after the explosion is even more depleted ( $90 \%$ of hydrogen, $10 \%$ of helium and other elements and compounds in traces), which, more or less, is the chemical composition of the rest of nebulae.

The information about the total quantity of the disintegrated visible matter can be found in the previous article (or (real data) $400 \mathrm{x}$ (factor 3 ) $=1200 \mathrm{x} \sim 100$ billion galaxies in the Universe x min. 8 M Sun > 8493 galaxies of the Milky Way size), an approximately real value of the disintegrated visible matter in the Universe caused by the explosions of stars. 


\section{Discussion}

\subsection{A Creation of Visible Matter}

That contemporary understanding of the Universe is seriously out of balance can be deducted from the facts of the Universe constantly expanding, gaining mass, from the omnipresent disintegration of the visible matter and the constantly ascending process of matter and system gathering (gravity coupled systems, small and large mergers and collisions of bodies, galaxies and clusters of galaxies). On one hand, enormous quantities of the visible matter get disintegrated every second, but on the other side, there is a constant growth of the visible matter, through objects and systems.

Table 5. The small and large mergers, collisions, gravitationally connected of object

Object

$\begin{array}{ll}1 & \text { Messier 66 } \\ 2 & \text { NGC 7479 } \\ 3 & \text { Arp 299 } \\ 4 & \text { Arp 87 } \\ 5 & \text { Arp 272 } \\ 6 & \text { MRC 1138-262 }\end{array}$

$7 \quad$ CL $1358+62$

$8 \quad \underline{R D} 1$

\section{Red shift}

0,002425

0,00794

0,010

0,023726

0,034239

2.156 the small and large mergers, collisions, gravitationally connected

M65 and NGC 3628

SN 1990U and SN2009jf

IC 694 and NGC 3690

NGC 3808A i NGC 3808B

NGC 6050 and IC 1179

It is formed from dozens of smaller galaxies that were seen in the process of merging the most distant galaxy merger discovered, as of 2008

0140 + 326 RD1
Distance M

ly

31

105

130

330

$494,13 \pm 55,89$

10600

11400

12000

Table 5. Object, the small and large mergers, collisions, gravitationally connected A disbalance is again noticeable in the following: "A chemical composition of a nebula is quite balanced; a fact which, by the way, follows the general composition of the Universe, which approximately consists of $90 \%$ of hydrogen atoms and almost all of the rest is helium $(\sim 10 \%)$ with oxygen, carbon, neon, nitrogen and other elements, which, put together, make two atoms per one thousand of them"

A chemical composition of stars: (Sun Photospheric composition (by mass): Hydrogen $73.46 \%$, Helium $24.85 \%$, Oxygen $0.77 \%$, Carbon $0.29 \%$, Iron $0.16 \%$, Neon $0.12 \% \ldots$ all heavier elements total $\sim 1,5-2 \%$ (There are only trace amounts of other elements, including oxygen, carbon, nitrogen, silicon, magnesium, neon, iron, and sulfur. These trace elements make up less than 0.1 percent of the mass of the Sun) (Helmenstine, 2018). there is again a significant discrepancy between their chemical compositions and the compositions of the remainders after the explosions of stars and also those of nebulae and the Universe.

Until now it has been discovered a bit more than 400 remainders of super novae in our galaxy (a total number of stars in our galaxy is 200-400 billion), which does not by far match the total mass of 3-5\% of interstellar matter in the Milky Way. A chemical composition of nebulae and stars tell us that the explosions of stars reduce the diversity of elements, existing on a star prior to the explosion. 
It is very important to say here that the diversity of a chemical composition of stars is significantly lower in the terms of quantity, ratio and complex atoms, than the ones of the objects that are in the orbit of a star. (Duckss, 2019).

The claims that complex elements are created in the explosions of stars and that they arrived to our planet, without having analyzed the chemical composition of all the objects in our system, are unrealistic. Uneven and different chemical compositions of the Sun and its orbiting objects deny such a hypothesis. Relating the existence of complex atoms to the remainders of the stars' explosions is incorrect, because the chemical composition of the remainders, left after a star has exploded, is in a total discrepancy with the composition of objects in our system and because, if that were the case, the chemical composition of all the objects would have been the same, which is disproved by the research and the evidence.

Quote: The object 67P/Churymov-Garasimenko, classified as a comet, has a lower density of all so-called gaseous planets. Although it is relatively close to Sun, its aggregate state is solid, so Philae could easily land on its surface. This fact clearly states that gaseous planets are solid (and solid/melted) objects with impressive atmospheres.

There are solid objects with even lower density: Pan $0,42 \mathrm{~g} / \mathrm{cm} 3$, Atlas $0,46 \mathrm{~g} / \mathrm{cm} 3$, Pandora $0,48 \mathrm{~g} / \mathrm{cm} 3$ - all of them the satellites of Saturn. Etc.

The objects that are closer to the central object possess a higher density (due to the higher tidal force effects), as well as the objects with bigger masses and higher temperatures of space (Ariel/Umbriel; Titania/Oberon; Proteus/Triton; Rhea/Iapetus; Galileo's satellites; Phobos/Deimos; internal/external planets; etc). Of course, it does not mean that all objects belong to this group. The very division of asteroids into $\mathrm{S}, \mathrm{M}$ and $\mathrm{V}$ type suggests a dramatical deviation. One part of objects becomes more dense in the beginning of their approach to the Sun (because volatile matter disappears and higher temperatures help the creation of the more complex elements). The other part of objects was created during the disintegration of objects (the internal - the higher density, and the external - the lower density), due to the collisions. In both cases a continuation of growth must be taken into consideration, as the lesser objects keep arriving to their surfaces. A certain portion of satellites also does not abide the strict law (density, mass, space temperature and distance to the central object), which implies the different past of these objects before they got captured by the central object. A part of it definitely belongs to the different composition of objects that constantly bombard satellites and other objects. It is unlikely that more dense asteroids from the asteroid belt would hit the outer objects, unlike the interior ones, because the gravitational force of Sun is dominant.

The conclusion would be that it is a very complex and dynamic pattern related to the processes of objects' creation - it is constantly moving and growing. The complexity of objects is related to the space temperature, the mass of an object and the total sum of tidal forces. Furthermore, the complexity is influenced by the position of an object related to the planet, Sun, as well as the asteroid belt. The important role also belongs to time when object got captured, for how long the object had been near Sun (perihelion) and at what distance. end quote. (Sedic, 2016).

The creation of complex elements is seen in the process of removing the volatile elements of the comets, which is violent and voluminous at the beginning. When a comet has made enough orbits around a star, the quantity of volatile elements in it is reduced and it turns into an asteroid. It should be pointed out that a chemical composition of a comet gets more complex with every turn around the Sun, which is at the end represented in the chemical composition of the asteroid. (Bardyn, 2017) 
The impossibility to relate the chemical compositions of planets and stars with the compositions of nebulae and interstellar material indicates that there is a process of creating new visible matter. That is particularly seen from the chemical composition of a material, which is outside the objects in the space. The first complex particle in the creation is hydrogen (in the atomic state), the fact demonstrated by the presence of this particle in nebulae, between objects and inside the Universe (90\%). During time, the creation of the other particles follows the ratio: helium $\sim 10 \%$ and all the other elements are only in traces, up to $2 \%$ maximum (Sun $\sim 1,7 \%$ ).

A greater diversity of all elements starts to appear when, due to the forces of attraction, the objects orbiting around a star start appearing in the orbits around the stars (high temperatures decompose complex atoms).

The greatest diversity is found on the objects (i.e., in their crust) that have a melted core, have an independent rotation and are mostly closer to a star. The creation of complex atoms takes place in the crust of such an objects, due to the pressure of the melted core on the crust, which itself is like a laboratory for the creation of complex atoms and compounds. A part of creation also takes place in the contact of the melted matter with water, atmosphere, ... This is seen on Mars, which has no melted core nor there are dynamic geological processes, necessary to create large quantities of complex atoms and compounds. Small quantities of hydrogen quickly migrate from Mars towards the Sun or get decomposed because of the radiation waves and they leave the planet with deserts and without water or compounds based on hydrogen.

\subsection{Processes Related to the Constant Ascending Process of Matter Gathering}

The process of matter gathering is seen on Earth and in the outer space. Matter gathers into nebulae, small and large objects, small and large systems. Sedic (2016) says that it can be deducted from the percussive craters on Earth and the other objects in our system.

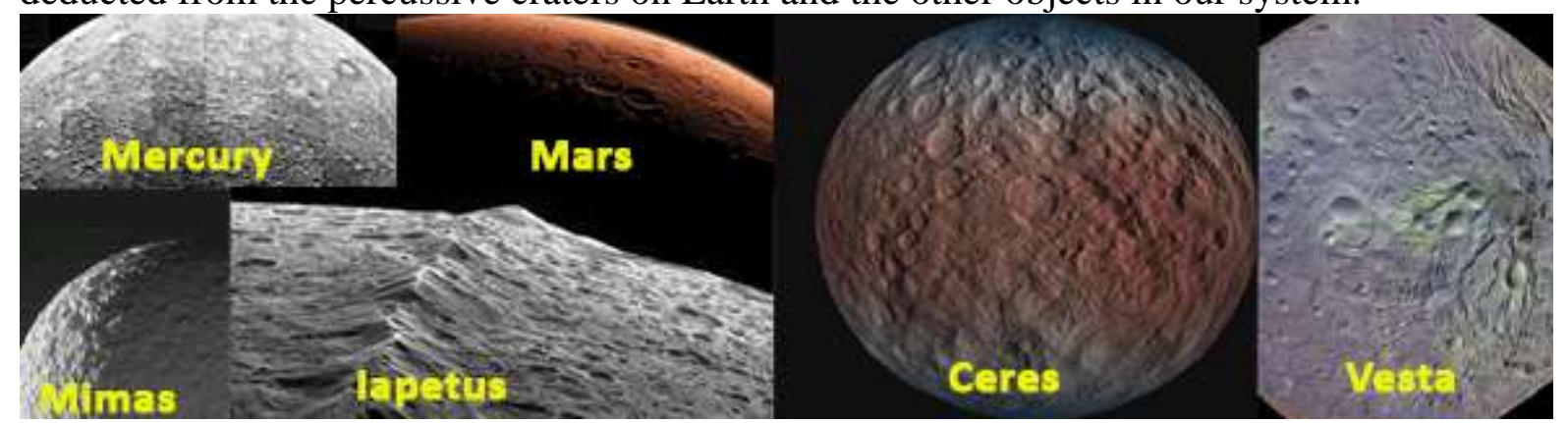

Figure 1. Percussive craters on some objects (NASA)

Percussive craters have covered completely such objects that lack atmosphere, independent rotation, that have a relatively solid surface and only minimal internal geological processes to remove the craters. A constant growth is presented by old craters, inside which new ones have appeared. Inside these new ones there are even newer ones... The frequency of such objects arriving to Earth (measured in their quantity, mass and the time interval in which they are appearing) makes it possible to conclude that the period of creating such reliefs on these objects is quite long and that it is a constant process. 


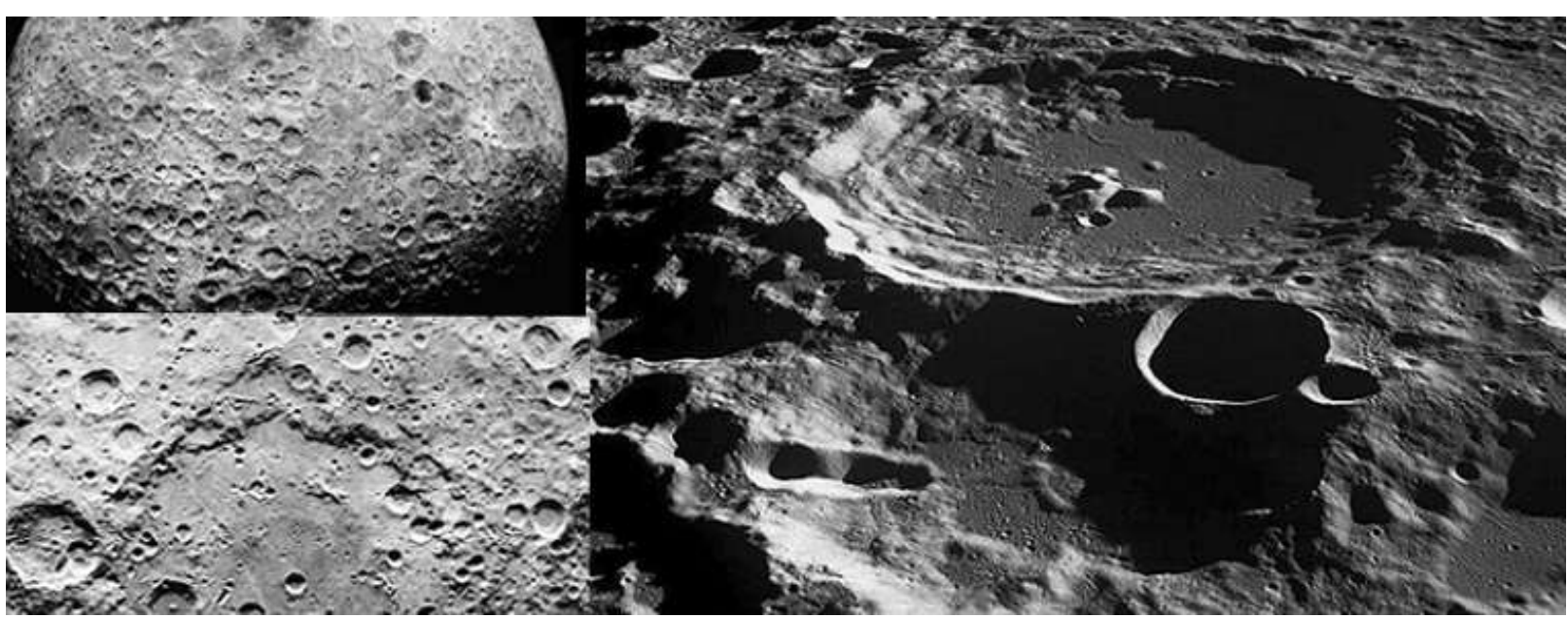

Figure 2. Craters (NASA)

The duration of process is seen from the daily arrival of the space material onto Earth (quantity estimates ranging from 50 to 300 tons per day (Duckss, 2019).

With the increase of an object's mass and also with the participation of tidal forces from the central object and the other objects, too, as well as the speed of rotation around its axis, such an object starts emitting the surplus of its own radiation, which is the indicator of a melted (hot) core being created (Jupiter, Neptune).

Table 6. Brown dwarf and planets, mass/temperature

\section{Mass up to $15 \mathrm{MJ} /(\mathrm{vs})$ Mass above $15 \mathrm{M}$}

Brown dwarf (\& planets) $\quad$ Mass of Jupiter Temperature ${ }^{\circ} \mathrm{K} \quad$ Planets orbit AU

1 ROXs 42Bb

9

$2 \underline{54 \text { Piscium B }}$ 9

3 DH Tauri b

50

$1.950 \pm 100$

157

4 ULAS J133553.45+113005.2 15 -31

$12 \quad 2.750$

330

5 OTS 44

11,5

$500-550$

6 Epsilon Indi $\mathrm{Ba}$ and $\mathrm{Bb}$

7 2MASS J2126-8140

8 Gliese 570

$40-60(28 \pm 7)$

$1.700-2.300$

Mass vs Mass

$\begin{array}{lllll}9 & \text { 2M 044144 } & 9.8 \pm 1.8 & 1.800 & 15 \pm 0.6 \\ 10 & \text { DT Virginis } & 8.5 \pm 2.5 & 695 \pm 60 & 1.168 \\ 11 & \text { Teide 1 } & 57 \pm 15 & 2.600 \pm 150 & \\ 12 & \text { Epsilon Indi Ba and Bb } & 40-60(28 \pm 7) & 1.300-1400 & (880-940) 1.500 \text { (between 2,1) } \\ 13 & \text { B Tauri FU } & 15 & 2.375 & 700 \\ 14 & \text { DENIS J081730.0-615520 } & 15 & 950 & \end{array}$

Table 6. Brown dwarf and planets (at a great distance), relationship: mass up to 15 $\mathrm{MJ} /(\mathrm{vs})$ mass above $15 \mathrm{M}$ and Mass vs Mass and temperature (Samuel, 2019). 
The core melting with the significant influence of tidal forces is seen on Venus, which is smaller than Earth and lacks its own rotation, but it has a significantly higher temperature and more active volcanic processes than Earth. A lack of mass is impossible to compensate with a rotation and tidal forces, which can be monitored on Mars, Mercury, Uranus, ... - these objects emit no significant radiation (Uran 1,06), at least they are less important than those incoming from the central object. The existence of melted core (i.e., matter) is the beginning of the process of creating hot objects from brown dwarfs to the largest stars and stars with a very fast rotation (,O“ type $\sim 0,00003 \%$ from the total number of stars in Milky Way).

A rotation of an object around its axis creates orbits for smaller objects and matter around a central object, creates also binary systems, globular clusters of stars, galaxies, clusters of galaxies, super clusters of galaxies, the Universe, Multiverse and, at the most, two systems more. When the objects that emit radiation (which creates light and heat in the collision with the visible matter) get diluted, the outer space and the visible matter that emits none of its own radiation have the temperature of $0^{\circ} \mathrm{K}$ and all of the processes either stop or become extremely slow.

One should always keep in mind that this is only one in the endless sequence of such or similar systems that exist in the Absolute zero.

Tidally locked objects (i.e., those that lack their own independent rotation) or those with an extremely slow rotation cannot create orbits, just as the objects with a rotation cannot create orbits around their poles (north - south).

\subsection{The Age of Objects and the Universe}

A constant growth or a constant matter gathering, in contemporary terms of understanding the age of Universe, is a very slow process without any form of sensationalism and ascribing supernatural abilities to the laws of physics (nature). As a starting point in determining the age of the Universe I will use the agreed age estimate for the asteroids and the materials from the Moon, which is about 4.5 billion of years. The quantity of matter, which is daily arriving to Earth, is 50 to 300 tons per day.

It needs to be mentioned that in certain phases growth has a different pace, which is also different in the whole volume of the Universe. The same goes for any object in a star system. For example, an object existing in an asteroid belt has a different growth pace than the one existing in a gaseous disk outside that belt, no matter be it internal or external objects.

When matter gets gathered into clouds (nebulae), the forces of attraction become stronger. The larger the object and the faster the rotation, the influence of the forces of attraction is more significant.

It would be much easier to determine the age of Earth if we were able to measure the age of melted matter. The rock, originated from lava, is 0 years old, equally today and 4 Gy ago (zircon from the Jack Hills Western Australia „Dashed line indicates $4.4 \mathrm{Ga}(\mathrm{y})$ apparent $207 \mathrm{~Pb} / 206 \mathrm{~Pb}$ age“ (Ge, 2018).

If we were to adapt the matter gathering to the growing mass (an asteroid with 4,5 Gy of age) and the daily arrival of matter to Earth, which is 50 to 300 tons per day, we would have the approximate result of $6 \times 10^{24}$ of years $\left(10^{24}\right.$ : in short scale : a septillion ; in long scale : a quadrillion of years). It should be mentioned here that larger objects ,steal“ matter $\left(\mathrm{H}_{2}\right.$ and $\left.\mathrm{He}\right)$ from smaller objects, which changes the approach in determining the age for each object. 
The diameter of the Universe is calculated to be about 13,7 G ly. (If ,the most distant objects in the universe are the galaxies GN-z11 13,39 G ly (billion light years), EGSY8p7 13,23 G ly, GRB 090423 13,18 G ly, etc.).

Table 7. the direction of the farthest galaxies within the Universe Galaxy Right ascension Declination Red shift Distance G

\begin{tabular}{|c|c|c|c|c|c|}
\hline & & & & & \\
\hline 1 & $\underline{\mathrm{HCM}-6 \mathrm{~A}}$ & $02^{\mathrm{h}} 39^{\mathrm{m}} 54.7^{\mathrm{s}}$ & $-01^{\circ} 33^{\prime} 32^{\prime \prime}$ & 6,56 & 12,8 \\
\hline 2 & SXDF-NB1006-2 & $02^{\mathrm{h}} 18^{\mathrm{m}} 56.5^{\mathrm{s}}$ & $-05^{\circ} 19^{\prime} 58.9^{\prime \prime}$ & 7,215 & 13,07 \\
\hline 3 & TN J0924-2201 & $09^{\mathrm{h}} 24^{\mathrm{m}} 19,92^{\mathrm{s}}$ & $-22^{\circ} 01 ' 41,5^{\prime \prime}$ & 5,19 & 12,523 \\
\hline 4 & UDFy-38135539 & $03^{\mathrm{h}} 32^{\mathrm{m}} 38.13^{\mathrm{s}}$ & $-27^{\circ} 45^{\prime} 53.9^{\prime \prime}$ & 8,6 & 13,1 \\
\hline 5 & A2744 YD4 & $00^{\mathrm{h}} 14^{\mathrm{m}} 24.927^{\mathrm{s}}$ & $-30^{\circ} 22^{\prime} 56.15^{\prime \prime}$ & 8,38 & 13,2 \\
\hline 6 & BDF-3299 & $22^{\mathrm{h}} 28^{\mathrm{m}} 12.26^{\mathrm{s}}$ & $-35^{\circ} 09^{\prime} 59.4^{\prime \prime}$ & 7,109 & 13,05 \\
\hline 7 & SSA22-HCM1 & $22^{\mathrm{h}} 17^{\mathrm{m}} 39.69^{\mathrm{s}}$ & $+00^{\circ} 13^{\prime} 48.6^{\prime \prime}$ & 5,47 & 12,7 \\
\hline 8 & $\begin{array}{l}\overline{\mathrm{EQ}} \\
\mathrm{J} 100054+023435\end{array}$ & $10^{\mathrm{h}} 00^{\mathrm{m}} 54.52^{\mathrm{s}}$ & $+2^{\circ} 34^{\prime} 35.17^{\prime \prime}$ & $\begin{array}{l}4,547(280.919 \\
\mathrm{km} / \mathrm{s})\end{array}$ & 12,2 \\
\hline 9 & $\begin{array}{l}\text { ULAS } \\
\text { J1120+0641 }\end{array}$ & $11^{\mathrm{h}} 20^{\mathrm{m}} 01.48^{\mathrm{s}}$ & $+06^{\circ} 41^{\prime} 24.3^{\prime \prime}$ & 7,085 & 13,05 \\
\hline 10 & $\begin{array}{ll}\text { ULAS } & \text { J1342 } \\
\underline{0928} & \end{array}$ & $13^{\mathrm{h}} 42^{\mathrm{m}} 08.10^{\mathrm{s}}$ & $+13^{\mathrm{h}} 42^{\mathrm{m}} 08.10^{\mathrm{s}}$ & 7,54 & 13,1 \\
\hline 11 & $\overline{\mathrm{GRB}} 090423$ & $09^{\mathrm{h}} 55^{\mathrm{m}} 33.08^{\mathrm{s}}$ & $+18^{\circ} 08^{\prime} 58.9^{\prime \prime}$ & 8,2 & 13 \\
\hline 12 & $\underline{\mathrm{IOK}-1}$ & $13^{\mathrm{h}} 23^{\mathrm{m}} 59.8^{\mathrm{s}}$ & $+27^{\circ} 24^{\prime} 56^{\prime \prime}$ & 6,96 & 12,88 \\
\hline 13 & $\overline{\mathrm{A} 1703} \mathrm{zD} 6$ & $13^{\mathrm{h}} 15^{\mathrm{m}} 01.0^{\mathrm{s}}$ & $+51^{\circ} 50^{\prime} 04^{\prime}$ & 7,054 & 13,04 \\
\hline 14 & $\underline{Q} 0906+6930$ & $09^{\mathrm{h}} 06^{\mathrm{m}} 30.75^{\mathrm{s}}$ & $+69^{\circ} 30^{\prime} 30.8^{\prime \prime}$ & 5,47 & 12,3 \\
\hline 15 & MACS0647-JD & $06^{\mathrm{h}} 47^{\mathrm{m}} 55.73^{\mathrm{s}}$ & $+70^{\circ} 14^{\prime} 35.8^{\prime \prime}$ & 10,7 & 13,3 \\
\hline
\end{tabular}

Table 7. the direction of the farthest galaxies within the Universe distance 12,2 -13,3 G $1 y^{\prime \prime}$ (Duckss, 2018). The Universe rotates at the speed of up to $30.000 \mathrm{~km} / \mathrm{s}$ [12] (which is far below contemporary data that do not consider that distance contributes to the increase in red spectrum). That speed is sufficient to create a disk-shaped form of the Universe.

Table 8. Red shift /distance

\begin{tabular}{l|l|l|l|}
\multicolumn{2}{c}{$\begin{array}{l}\text { Galaxy, Cluster galaxy, } \\
\text { Supercluster }\end{array}$} & Red shift (z) & Distance M ly \\
\hline 1 & Leo_Cluster & 0,022 & 368,6 \\
\hline 2 & ARP 87 & 0,023726 & 330 \\
\hline 3 & Abell 2152 & 0,041 & 551 \\
\hline 4 & Hydra_Cluster & 0,0548 & 190,1 \\
\hline 5 & Abell 671 & 0,0502 & 600 \\
\hline 6. & Abell 1060 & 0,0548 & 190,1 \\
\hline 7 & Abell_1991 & 0,0587 & 812 \\
\hline 8 & Corona Borealis Supercluster & 0,07 & 946 \\
\hline 9 & Laniakea Supercluster & 0,0708 & 250 \\
\hline 10 & Abell 2029 & 0,0767 & 1063 \\
\hline 11 & Abell 383 & 0,1871 & 2485 \\
\hline 12 & Abell 520 & 0,2 & 2645 \\
\hline
\end{tabular}


Budapest International Research in Exact Sciences (BirEx) Journal

Volume 1, No 4, October 2019, Page: 12-24

e-ISSN: 2655-7827 (Online), p-ISSN: 2655-7835 (Print)

www.bircu-journal.com/index.php/birex

emails: birex.journal@gmail.com

birex.journal.qa@gmail.com

\begin{tabular}{|l|l|l|l|}
\hline 13 & Abell_222(3) & 0,211 & 2400 \\
\hline 14 & Saraswati Supercluster & 0,28 & 4000 \\
\hline 15 & Bullet Cluster & 0,296 & 3700 \\
\hline 16 & Abell 2744 & 0,308 & 3982 \\
\hline 17 & CID-42 & 0,359 & 3900 \\
\hline 18 & Abell_370 & 0,375 & 4775 \\
\hline 19 & $\underline{\text { 3C_295 }}$ & 0,464 & 4600 \\
\hline 20 & Musket Ball Cluster & 0,53 & 700 \\
\hline 21 & Abell 754 & 0,542 & 760 \\
\hline 22 & MACS J0025.4-1222 & 0,586 & 6070 \\
\hline 23 & Phoenix Cluster & 0,597 & 5700 \\
\hline 24 & $\underline{\text { RX J1131-1231 }}$ & 0,658 & 6050 \\
\hline 25 & ACT-CL J0102-4915 & 0,87 & 4000 \\
\hline 26 & Lynx Supercluster & $1,26,1,27$ & 12000 \\
\hline 27 & Twin Quasar & 1,413 & 8700 \\
\hline 28 & $\underline{\text { XMMXCS_2215-1738 }}$ & 1,45 & 10000 \\
\hline 29 & Einstein Cross & 1,695 & 8000 \\
\hline 30 & TON 618 & 2,219 & 10,400 \\
\hline 31 & EQ J100054+023435 & 4,547 & 12200 \\
\hline 32 & $\underline{\text { Z8 GND 5296 }}$ & $7.5078 \pm 0.0004$ & 13100 \\
\hline 32 & A2744 YD4 & 8,38 & 13200 \\
\hline 33 & $\underline{\text { UDFy-38135539 }}$ & 8,6 & 13100 \\
\hline 34 & $\underline{\text { GRB 090429B }}$ & 9,4 & 13140 \\
\hline 35 & $\underline{\text { Abell 1835 IR1916 }}$ & 10,0 & 13200 \\
\hline & & & \\
\hline
\end{tabular}

Table 8. As the red spectrum increases, the distance between objects decreases, increases (faster or slower than "expected") or remains similar. Quote: If two or more systems merge or are in some other form of interaction, the detected redshift in all of these systems should not be interpreted exclusively as a result of distancing the systems. A part of these systems is getting closer to an observer and a blueshift should be detected there, but it is not. With the increase of distance, the intensity of waves is decreased - the consequence of which is the increase in red spectrum, independently of the object being distanced away or getting closer to an observer.
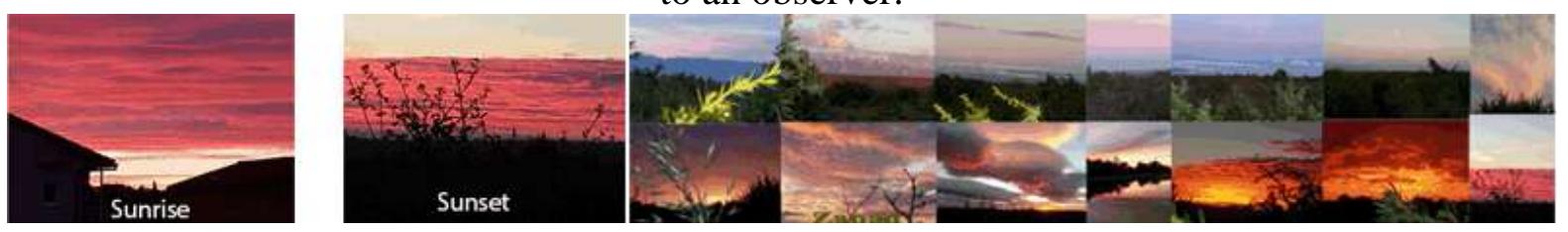

Figure 3. A red color before sunrise and after sunset; to the east (up) and to the west (down) at sunset (Zadar, Croatia)

A red color is directly related to the decrease of wave intensity from the emitting object. On the images, the Sun is behind the horizon. After a certain distance the weakening of radiation intensity overcomes the speed of the system getting closer to the observer and after that distance it gets impossible to detect the blueshift. In the processes of getting closer, merger and collisions of galaxies and clusters of galaxies there is only the blueshift among these systems, although the redshift is detected, because of the low wave intensity. Nowadays, the 
blueshift is not detected above $70 \mathrm{M}$ ly. An exact example is the appearance of a red moon. Moon gets red when it is in the shadow of Earth. The waves from Sun do not reach Moon then.

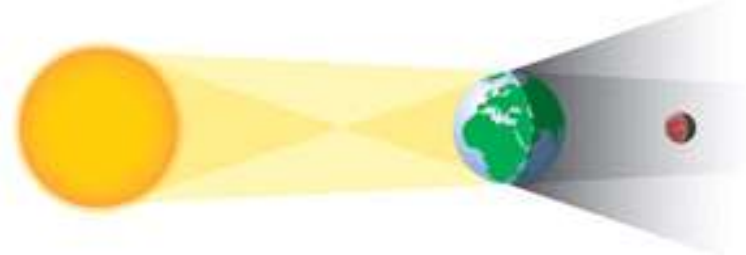

Figure 4. Red Moon, a display of the process. end quote.

To achieve a disk-shaped form of a system, it takes, besides the speed of rotation, a large number of turns around some axis. The approximate diameter of the Universe is about 27 Gly ( $r$ is $\sim 13,7 \mathrm{~Gy}$ ). Besides the process of constant growth, the processes of disintegration and the creation of matter should also be included in the calculations about the Universe. With an approximate speed of rotation reaching $10 \%$ of the speed of light, the Universe makes a single turn in $\sim 860 \mathrm{~Gy}$. This number needs to be multiplied with a very large number of turns around its axis

\section{Conclusion}

Particles are disintegrated by force due to the percussive waves from stars to the atmospheres of the objects in their orbits, due to objects' collisions, due to cyclones in the objects' polar regions, due to explosions of stars and due to our particle colliders.

The creation of the visible matter is seen in the increase of mass of the Universe and its chemical composition ( $\mathrm{H} \sim 90 \%$; $\mathrm{He} \sim 10 \%$, the rest of the elements are in traces, up to $2 \%$ ).

A constant, ascending growth (the consolidation of objects and systems) is registered as the arrival of matter to the formed objects, which is proved by the millions of percussive craters on the objects, by the processes of collisions, merger and interaction of objects, galaxies and the clusters of galaxies.

The distance between the objects in the outer space creates a red shift; after some distance $(=70$ Gly), no matter whether galaxies are approaching to the observer or not, which is concluded from the collisions, mergers and interactions of galaxies and the rotations of galaxy clusters and their collisions, mergers and the creation of super clusters.

The age of Earth and other objects is determined by the time needed to gather matter, influenced also by the constant forces of attraction. Every object has a different growth pace, which depends on its position in a system or the position of the system in the Universe.

The age of the Universe is determined by the constant growth, creation and disintegration of matter and the time needed to gather a whole system with a disk-shaped form, due to a relatively fast rotation.

\section{References}

Bardyn, A. (2017). Carbon-rich dust in comet 67P/Churyumov-Gerasimenko measured by COSIMA/Rosetta. Monthly Notices of the Royal Astronomical Society, S712-S722. Duckss, W. (2019). Učinci Rotacije Oko Osi Na Zvijezde, Galaksiju I Rotaciju Svemira. International Journal of Sciences, 76-97. 
Duckss, W. (2019). When Occurring Conditions for the Emergence of Life and a Constant Growth, Rotation and its Effects, Cyclones, Light and Redshift in Images. International Journal of Sciences, 34-46.

Duckss, W. (2019). Why do Hydrogen and Helium Migrate from Some Planets and Smaller Objects?. Intellectual Archive.

Duckss, W. (2018). The Processes Which Cause the Appearance of Objects and Systems. American Journal of Astronomy and Astrophysics, 72-80.

Ge, R. (2018). A 4463 Ma apparent zircon age from the Jack Hills (Western Australia) resulting from ancient $\mathrm{Pb}$ mobilization. Geology.

Helmenstine, A. M. (2018), What Is the Sun Made Of? Table of Element Composition. https://www.thoughtco.com/element-composition-of-sun-607581

John S. M. (2019). Nebula astronomy, Encyclopædia Britannica. https://www.britannica.com/science/nebula

John, E. (1979). A New Sun: The Solar Results From Skylab, by, NASA SP-402. Stanford Solar Center. http://solar-center.stanford.edu/vitalstats.html

Lagzi, I., Mészáros, R., Gelybó, G., Leelőssy, Á. (2013). Atmospheric Chemistry. Eötvös Loránd University. http://www.eltereader.hu/media/2014/04/Atmospheric_Chemistry_READER.pdf

Niemann, H. B. et al. (2005). The abundances of constituents of Titan's atmosphere from the GCMS instrument on the Huygens probe. Nature, 779-784.

Samuel, A. F. (2019). On the Performance of Transmuted Logistic Distribution: Statistical Properties and Application. Budapest International Research in Exact Sciences (BirEx) Journal, 26-34.

Sedic, S. (2016). Why there are differences in structure of the objects in our system. International Journal of Scientific \& Engineering Research, 408-422.

Street, J. C., \& Stevenson, E. C. (1937). New Evidence for the Existence of a Particle of Mass Intermediate Between the Proton and Electron. Physical Review Journals Archive.

Zigta, B. (2019). Thermal Radiation, Chemical Reaction and Viscous Dissipation Effects on Unsteady MHD Flow of Viscoelastic Fluid Embedded in a Porous Medium. Budapest International Research in Exact Sciences (BirEx) Journal, 35-57.

Williams, D. R. (2010). Mars Fact Sheet. National Space Science Data Center. NASA. https://web.archive.org/web/20100612092806/http:/nssdc.gsfc.nasa.gov/planetary/facts heet/marsfact.html

Williams, D. R. (2016). Saturn Fact Sheet. NASA. https://web.archive.org/web/20170717071200/https:/nssdc.gsfc.nasa.gov/planetary/fact sheet/saturnfact.html

Yukawa, H. (1935). On the Interaction of Elementary Particles. http://web.ihep.su/dbserv/compas/src/yukawa35/eng.pdf 